\title{
The binding site for neohesperidin dihydrochalcone at the human
} sweet taste receptor

\author{
Marcel Winnig ${ }^{\dagger 14}$, Bernd Bufe ${ }^{\dagger 1,5}$, Nicole A Kratochwil ${ }^{2}$, Jay P Slack ${ }^{3}$ and \\ Wolfgang Meyerhof*1
}

\author{
Address: ${ }^{1}$ German Institute of Human Nutrition Potsdam-Rehbruecke, Department of Molecular Genetics, Arthur-Scheunert Allee 114-116, 14558 \\ Nuthetal, Germany, ${ }^{2}$ Pharmaceuticals Division, F. Hoffmann-La Roche Ltd, Grenzacherstrasse 124, 4070 Basel, Switzerland, ${ }^{3}$ Givaudan Flavors \\ Corporation, 1199 Edison Drive, Cincinnati, 45216 Ohio, USA, ${ }^{4}$ Axxam, San Raffaele Biomedical Science Park, via Olgettina 58, 20132 Milan, \\ Italy and ${ }^{5}$ Department of Physiology, University of Saarland School of Medicine, 66421 Homburg/Saar, Germany \\ Email: Marcel Winnig - marcel.winnig.mw@axxam.com; Bernd Bufe - bernd.bufe@uks.eu; \\ Nicole A Kratochwil - nicole_a.kratochwil@roche.com; Jay P Slack - jay.slack@givaudan.com; Wolfgang Meyerhof* - meyerhof@dife.de \\ * Corresponding author †Equal contributors
}

Published: 12 October 2007

BMC Structural Biology 2007, 7:66 doi:10.1/86/1472-6807-7-66

This article is available from: http://www.biomedcentral.com/l472-6807/7/66

(C) 2007 Winnig et al; licensee BioMed Central Ltd.

This is an Open Access article distributed under the terms of the Creative Commons Attribution License (http://creativecommons.org/licenses/by/2.0), which permits unrestricted use, distribution, and reproduction in any medium, provided the original work is properly cited.
Accepted: 12 October 2007
Received: 7 June 2007

\begin{abstract}
Background: Differences in sweet taste perception among species depend on structural variations of the sweet taste receptor. The commercially used isovanillyl sweetener neohesperidin dihydrochalcone activates the human but not the rat sweet receptor TASIR2+TASIR3. Analysis of interspecies combinations and chimeras of rat and human TASIR2+TASIR3 suggested that the heptahelical domain of human TASIR3 is crucial for the activation of the sweet receptor by neohesperidin dihydrochalcone.

Results: By mutational analysis combined with functional studies and molecular modeling we identified a set of different amino acid residues within the heptahelical domain of human TASIR3 that forms the neohesperidin dihydrochalcone binding pocket. Sixteen amino acid residues in the transmembrane domains 2 to 7 and one in the extracellular loop 2 of hTASIR3 influenced the receptor's response to neohesperidin dihydrochalcone. Some of these seventeen residues are also part of the binding sites for the sweetener cyclamate or the sweet taste inhibitor lactisole. In line with this observation, lactisole inhibited activation of the sweet receptor by neohesperidin dihydrochalcone and cyclamate competitively, whereas receptor activation by aspartame, a sweetener known to bind to the $\mathrm{N}$-terminal domain of TASIR2, was allosterically inhibited. Seven of the amino acid positions crucial for activation of hTASIR2+hTASIR3 by neohesperidin dihydrochalcone are thought to play a role in the binding of allosteric modulators of other class C GPCRs, further supporting our model of the neohesperidin dihydrochalcone pharmacophore.

Conclusion: From our data we conclude that we identified the neohesperidin dihydrochalcone binding site at the human sweet taste receptor, which overlaps with those for the sweetener cyclamate and the sweet taste inhibitor lactisole. This readily delivers a molecular explanation of our finding that lactisole is a competitive inhibitor of the receptor activation by neohesperidin dihydrochalcone and cyclamate. Some of the amino acid positions crucial for activation of hTASIR2+hTASIR3 by neohesperidin dihydrochalcone are involved in the binding of allosteric modulators in other class C GPCRs, suggesting a general role of these amino acid positions in allosterism and pointing to a common architecture of the heptahelical domains of class C GPCRs.
\end{abstract}




\section{Background}

Genetic, anatomical and functional studies provide compelling evidence that the vast majority of sweet taste perception is mediated by G-protein coupled receptors (GPCR) of the TAS1R-gene family, which comprises the members TAS1R1-3 [1-6]. TAS1Rs belong to the class C GPCRs and are distantly related to the calcium sensing receptor, metabotropic glutamate receptors, V2R pheromone receptors, and $\mathrm{GABA}_{\mathrm{B}}$ receptors [3]. In situ hybridization studies revealed that TAS1R3 is coexpressed with TAS1R1 or TAS1R2 in taste receptor cells $[5,6]$. This observation suggests that the functional receptor, like other class C GPCRs [7], may be a heteromer of two subunits. Indeed, functional assays revealed that the combination of TAS1R1+TAS1R3 is activated by umami tasting compounds, while TAS1R2+TAS1R3 responds to sweeteners $[4,5,8]$.

The human sweet taste receptor is sensitive to the sweet proteins thaumatin, brazzein and monellin, the artificial sweeteners aspartame and cyclamate as well as to the sweet inhibitor lactisole whereas its rodent homolog is not [4]. This is in line with corresponding variations in sweet perception across species [9-11]. Studies with chimeric receptors revealed that replacement of the large $\mathrm{N}$ terminal extracellular domain of rat Tas1r2 by its human counterpart created a receptor that responded to aspartame and neotame when coexpressed with hTAS1R3. Additional mutations in the N-terminal domain of human TAS1R2 impaired the activation of the sweet taste receptor by aspartame, thus suggesting that the N-terminal part of TAS1R2 is involved in the binding of these sweeteners $[12,13]$. Similar studies showed that amino acids in the cysteine-rich region of human TAS1R3 that connects the $\mathrm{N}$-terminal extracellular domain to the segment containing the heptahelical domain determines the response to sweet proteins such as brazzein and monellin [12]. Conversely, replacement of the heptahelical domain of rat Tas1r3 by the corresponding part of the human receptor led to a chimera that responded to lactisole and cyclamate when coexpressed with rat Tas1r2 [13]. This suggests that the binding sites for cyclamate and lactisole are located in the heptahelical domain of hTAS1R3. Indeed, mutational analysis in combination with molecular modeling studies of the heptahelical domain of TAS1R3 revealed that the sweet inhibitor lactisole and the sweetener cyclamate have overlapping binding sites in the heptahelical domain of the human TAS1R3 subunit $[14,15]$. Recently, analysis of rat-human sweet taste receptor chimeras revealed that the heptahelical domain of hTAS1R3 is also crucial for the activation by the sweetener neohesperidin dihydrochalcone (NHDC) [16]. NHDC is added to various foods and beverages as a low caloric sweetener [17], but its use is limited by some unwanted sensory properties such as a delayed onset and a long lin- gering menthol-licorice like sweetness $[18,19]$. Thus, a detailed molecular understanding of the interactions of the sweet receptor with NHDC may contribute to the rational design of analogues with improved sensory properties. We therefore investigated the binding pocket of neohesperidin dihydrochalcone at the human sweet taste receptor.

\section{Results \\ Analysis of receptor chimeras reveals that NHDC requires the human TASIR3 heptahelical domain}

The isovanillyl compound NHDC (Fig. 1A) activates the human but not the rodent sweet taste receptor (Fig. 1B). In order to elucidate the putative binding site for NHDC, we cotransfected mixtures of plasmid DNAs for the rat and human TAS1R2 and TAS1R3 receptor subunits in HEK293T cells stably expressing the chimeric G-protein G16Gust44 and measured cellular calcium responses by fluorometry following bath application of various sweet tasting compounds. We found that NHDC elicited calcium responses in cells transfected with human TAS1R3 and rat Tas1r2 cDNA, whereas it did not in cells transfected with the opposite combination (Fig. 1C). By replacing the heptahelical domain of rat Tas1r3 with that of human TAS1R3 we produced a chimeric receptor that was sensitive for NHDC when coexpressed with rat Tas1r2 (Fig. 1C). In marked contrast, all receptors that contain the entire rat Tas1r3 or its heptahelical domain did not respond to NHDC (Fig. 1C). Notably, although the amplitudes and the response pattern of the receptor chimaeras varied (for details see Ref. 13, supplement) we can demonstrate that all tested receptor chimeras were functional because they could be activated by at least one sweetener (Fig. 1C, D). These results clearly indicate that the heptahelical domain of TAS1R3 appears to be crucially involved in the activation of the sweet receptor by NHDC.

\section{Receptor activation by NHDC is competitively inhibited by lactisole}

Recently, it has been shown that the binding sites for the sweetener cyclamate and the sweet inhibitor lactisole overlap in the heptahelical domain of TAS1R3 $[14,15]$. We therefore reasoned that the NHDC binding site might also overlap with that for lactisole. In this case one would expect that lactisole competitively inhibits the activation of hTAS1R2/hTAS1R3 by NHDC and cyclamate. In contrast, receptor activation by sweeteners that bind to other sites should be allosterically inhibited. To verify this assumption we recorded concentration-response curves for NHDC, cyclamate, acesulfame $\mathrm{K}$ and aspartame in the presence of different concentrations of lactisole in cells expressing hTAS1R2/hTAS1R3 (Fig. 2).

In line with these expectations lactisole did not alter the $\mathrm{EC}_{50}$ values for acesulfame $\mathrm{K}$ but reduced the signal ampli- 
A neohesperidin dihydrochalcone

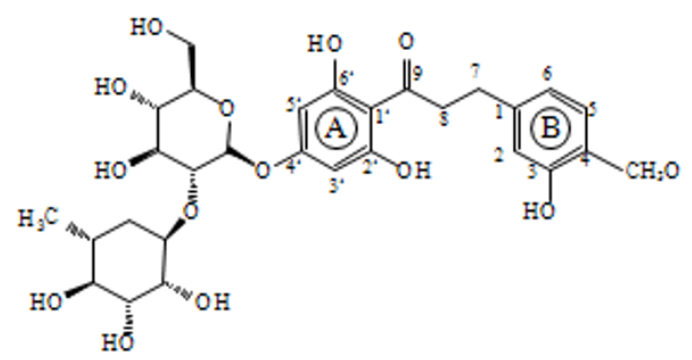

B
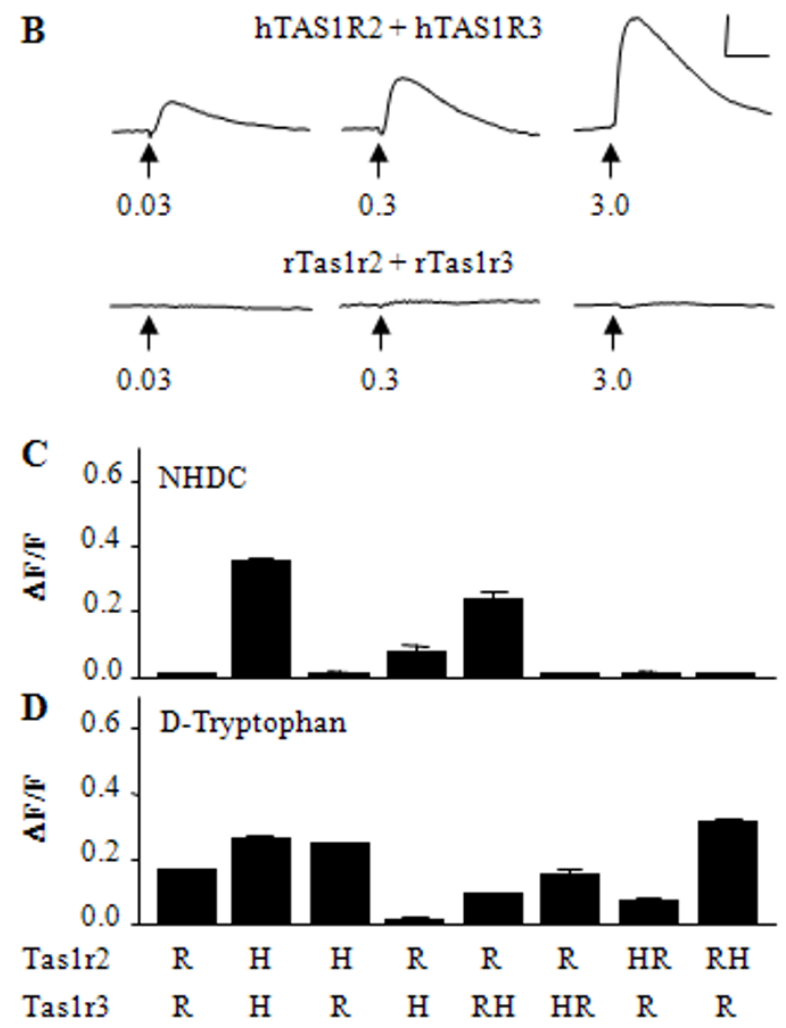

Figure I

Chemical structure and calcium responses elicited by neohesperidin dihydrochalcone. (A) Structure of neohesperidin dihydrochalcone. Numbers denote carbon atom positions. (B) Representative calcium traces elicited upon stimulation with different concentrations of neohesperidin dihydrochalcone (NHDC) in HEK293T-G I6Gust44 cells cotransfected with DNA for hTASIR2/hTASIR3. (C,D) Calcium responses of cells cotransfected with DNA for different rat and human TASIR subunits or chimeras upon stimulation with I mM NHDC (C), or 30 mM D-Tryptophan (D). R, rat receptor subunit; $H$ human receptor subunit; $H R$, receptor chimera comprising the $\mathrm{N}$-terminal extra cellular domain of the human receptor subunit fused to the corresponding heptahelical domain of the rat; $\mathrm{RH}$, comprising the $\mathrm{N}$-terminal extracellular domain of the rat receptor subunit fused to the corresponding heptahelical domain of the human receptor.
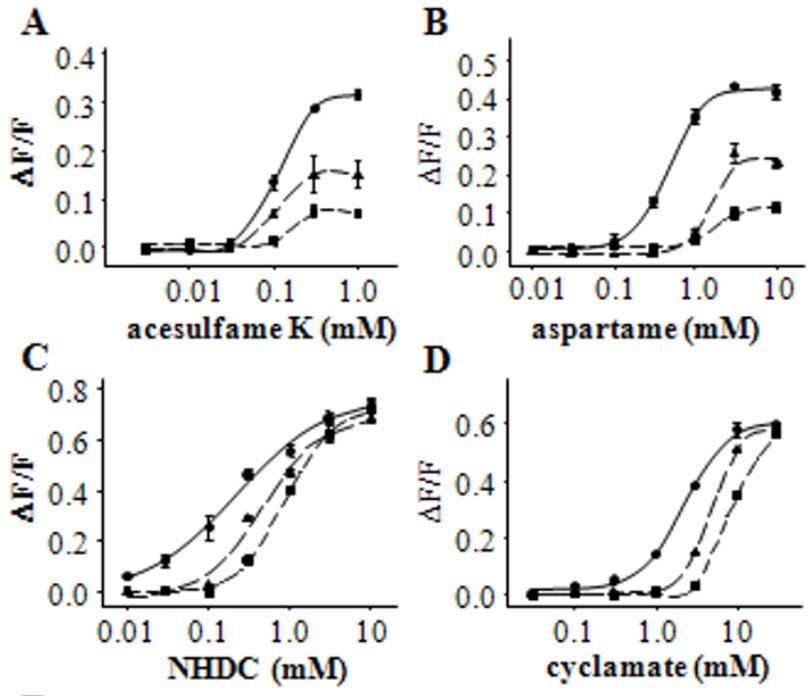

\begin{tabular}{l|ccc}
$\mathbf{E}$ & \multicolumn{3}{|c}{$\mathrm{EC}_{50}(\mathrm{mM})$ at } \\
sweetener & $0.0 \mu \mathrm{M}$ & $\begin{array}{c}50 \mu \mathrm{M} \\
\text { lactisole }\end{array}$ & $100 \mu \mathrm{M}$ \\
\hline acesulfame K & $0.12 \pm 0.04$ & $0.14 \pm 0.03$ & $0.17 \pm 0.03$ \\
aspartame & $0.5 \pm 0.1$ & $1.1 \pm 0.1$ & $1.4 \pm 0.3$ \\
NHDC & $0.2 \pm 0.1$ & $0.5 \pm 0.1$ & $0.9 \pm 0.1$ \\
cyclamate & $2.2 \pm 0.3$ & $4.9 \pm 0.3$ & $7.4 \pm 1.8$
\end{tabular}

Figure 2

Inhibition of hTASIR2/hTASIR3 by lactisole. (A-D) Concentration responses of cells transfected with hTASIR2hTASIR3 DNAs to sweetners mixed with different lactisole concentrations. No lactisole present (filled circles, solid line), $50 \mu \mathrm{M}$ lactisole (filled triangles up, dashed line), and $100 \mu \mathrm{M}$ lactisole (filled squares, dash-dotted line). (E) $\mathrm{EC}_{50}$ values for acesulfame $\mathrm{K}$, aspartame, NHDC, and cyclamate in the absence or presence of $50 \mu \mathrm{M}$ and $100 \mu \mathrm{M}$ lactisole.

tudes up to $75 \%$ (Fig. 2A, E), suggesting that it is an allosteric inhibitor of acesulfame K. Likewise, lactisole diminished the signal amplitudes induced by aspartame (Fig. 2B), which is consistent with an allosteric inhibition of aspartame-mediated receptor activation. Interestingly, in case of aspartame, the pronounced reductions in signal amplitudes were accompanied by a right-shift in the $\mathrm{EC}_{50}$ values (Fig. 2B, E). This may be due to an influence of lactisole on the aspartame binding site by negative cooperativity [20]. In contrast, lactisole did not diminish the signal amplitudes elicited by NHDC or cyclamate (Fig. $2 \mathrm{C}, \mathrm{D})$. Instead, lactisole clearly increased the $\mathrm{EC}_{50}$ values of these compounds fourfold (Fig. 2E). This observed competitive inhibition suggests that the binding sites for lactisole, cyclamate and NHDC overlap in the heptahelical domain of human TAS1R3. Interestingly, we observed 
slope changes for two of the four tested compounds. While Aspartame and Acesulfam $\mathrm{K}$ had constant Hill slope of $\sim 2$ the Hill coefficient for NHDC in the presence of $0.0 \mu \mathrm{M}, 50 \mu \mathrm{M}$ or $100 \mu \mathrm{M}$ lactisole was $0.8,1.2$ and 1.5, respectively. The calculated Hill coefficient for cyclamate was 1.6, 2.5 and 2.7, respectively. This may be best explained by allosteric effects and may therefore indicate the existence of additional interaction sites either for lactisole or for the sweeteners [21].

\section{NHDC interacts with residues of the lactisole and cyclamate binding site}

Next, we investigated which amino acids of the binding sites for lactisole or cyclamate are critical determinants for receptor activation by NHDC. Therefore, we analyzed the

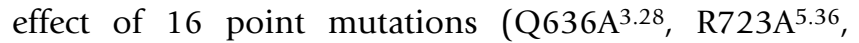

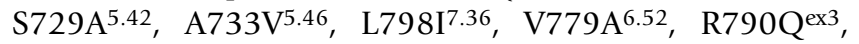

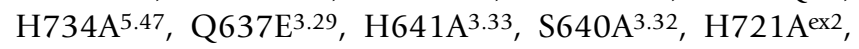
F730L 5.43 , W775A 6.48, F778A $^{6.51}$, L782A $^{6.55}$ ) in the heptahelical domain of hTAS1R3 that have previously been shown to influence receptor sensitivity to cyclamate or lactisole $[14,15]$ on the sweet receptor's response to NHDC. The effects were measured by recording calcium responses in HEK293T-G16Gust44 cells coexpressing hTAS1R2 and the various hTAS1R3 mutants. Cells

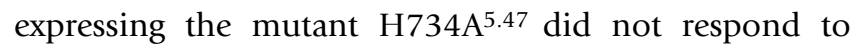
NHDC, cyclamate, aspartame or acesulfame K (Additional file 1). We assumed that this mutation generally impairs the function of the receptor and excluded it from further analyses. Seven receptor mutants (Q636A 3.28 , R723A5.36, S729A ${ }^{5.42}$, A733V5.46, V779A6.52, R790Q ${ }^{\text {ex3, }}$ $\mathrm{L}^{798 \mathrm{I}^{7.36}}$ ) showed $\mathrm{EC}_{50}$ values for NHDC similar to that of the wild type receptor (Table 1, normal script, Additional file 2), suggesting that these residues have no impact on the action of NHDC on the sweet receptor. The eight remaining TAS1R3 mutants, Q637E 3.29 , S640A 3.32 , H641A 3.33 , H721A ex2, F730L 5.43 , W775A 6.48 , F778A ${ }^{6.51}$,

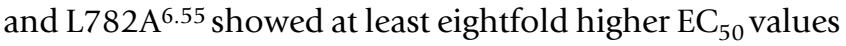
for NHDC than the wild type receptor (Table 1, bold script, Additional file 2). The decreased sensitivities of these eight mutant receptors were specific for NHDC since their $\mathrm{EC}_{50}$ values for aspartame were not altered (Table 1). Additionally, immunocytochemical analysis revealed similar expression patterns for all examined TAS1R3 receptor variants (Additional file 3 ). Thus, the increased $\mathrm{EC}_{50}$ values suggest that Q6373.29, H6413.33, S6403.32, $\mathrm{H} 721^{\mathrm{ex} 2}$, F7305.43, W7756.48, F7786.51, L782 ${ }^{6.55}$ are important for the activation of the sweet taste receptor by NHDC.

\section{Modeling of the NHDC binding site predicts interactions with additional residues in the heptahelical domain of TASIR3}

To further elucidate the binding mode for NHDC, an alignment of the seven transmembrane helices of the hTAS1R3 with the transmembrane helices of bovine rhodopsin was performed (Additional file 4). Amino acids were extracted at helix positions critical for binding of retinal and then combined in a one dimensional amino acid vector, called ligand pocket vector (LPV) [22]. Amino acids in the TAS1R3 LPV were considered as likely candidates to affect binding of NHDC. Classical homology models imply to have the quality of an X-ray crystal structure and therefore overstress specific, in detail unknown,

Table I: Effects of various mutations on the activation of the sweet taste receptor by neohesperidin dihydrochalcone (NHDC) and aspartame (ASP)

\begin{tabular}{|c|c|c|c|c|}
\hline variant & $\mathrm{NHDC} \mathrm{EC}_{50}(\mathrm{mM})$ & $\begin{array}{c}\text { NHDC maximal signal } \\
(\% \text { of } w t)\end{array}$ & ASP EC $\mathrm{EC}_{50}(\mathrm{mM})$ & $\begin{array}{c}\text { ASP maximal signal } \\
(\% \text { of } w t)\end{array}$ \\
\hline wt & $0.1 \pm 0.06$ & 100 & $\mathrm{I} .3 \pm 0 . \mathrm{I}$ & 100 \\
\hline Q636A 3.28 & $0.3 \pm 0.2$ & $61 \pm 29$ & $1.1 \pm 0.1$ & $70 \pm 18$ \\
\hline Q637E3.29 & $1.5 \pm 0.4$ & $51 \pm 13$ & $1.7 \pm 0.2$ & $51 \pm 10$ \\
\hline$S 640 A^{3.32}$ & $0.8 \pm 0.2$ & $63 \pm 18$ & $1.4 \pm 0.5$ & $61 \pm 40$ \\
\hline H64 I A 3.33 & $1.2 \pm 0.4$ & $57 \pm 22$ & $1.8 \pm 0.4$ & $79 \pm 70$ \\
\hline H72 I A ex2 & $0.8 \pm 0.3$ & $66 \pm 10$ & $1.6 \pm 0.3$ & $60 \pm 40$ \\
\hline$R 723 A^{5.36}$ & $0.2 \pm 0.1$ & $69 \pm 71$ & $1.5 \pm 0.5$ & $68 \pm 50$ \\
\hline$S 729 A^{5.42}$ & $0.2 \pm 0.1$ & $80 \pm 16$ & $0.9 \pm 0.4$ & $72 \pm 20$ \\
\hline F730L 5.43 & $0.9 \pm 0.1$ & $70 \pm 18$ & $1.7 \pm 0.4$ & $87 \pm 27$ \\
\hline A733V5.46 & $0.1 \pm 0.02$ & $133 \pm 8$ & $1.1 \pm 0.3$ & $92 \pm 80$ \\
\hline $\mathrm{H} 734 \mathrm{~A}^{5.47}$ & n.f. & -- & n.f. & -- \\
\hline W775A 6.48 & $>3$ & $30 \pm 41$ & $1.9 \pm 0.2$ & $20 \pm 30$ \\
\hline F778A 6.51 & $1.5 \pm 0.4$ & $50 \pm 18$ & $1.2 \pm 0.1$ & $80 \pm 80$ \\
\hline V779A 6.52 & $0.2 \pm 0.1$ & $88 \pm 14$ & $1.2 \pm 0.3$ & $52 \pm 10$ \\
\hline L782A 6.55 & $>3$ & $50 \pm I I$ & $1.7 \pm 0.3$ & $95 \pm 90$ \\
\hline R790Q ${ }^{\mathrm{ex} 3}$ & $0.1 \pm 0.06$ & $77 \pm 15$ & $0.9 \pm 0.4$ & $71 \pm 30$ \\
\hline L798I7.36 & $0.2 \pm 0.2$ & $66 \pm 26$ & $0.9 \pm 0.4$ & $81 \pm 5$ \\
\hline
\end{tabular}

Receptor variants that affect the NHDC response are shown in bold. Values are given as average over at least three independent experiments \pm SD. n.f., no response to any tested sweetener. $(>), E_{50}$ not calculable because saturation is not reached (Additional file 2 ). 
rotamer conformations of the amino acids. Despite of the substantial progress in building homology models and independently of the level of sophistication of the techniques used, there always remains the risk of a mismatch between optimized rotamers and ligand binding requirements, leading to the rejection of ligands in docking experiments. We therefore designed a simple TM binding pocket pharmacophore (Fig. 3A) to avoid atomic details tending to mask this inherent uncertainty and to concentrate on the general topology of the binding site and the approximate relative orientation of charged, donor/acceptor and neutral side chains. Only amino acid residues are shown which were identified as important for the action of NHDC by site-directed mutagenesis. NHDC was manually docked into the pharmacophore. For the docking process in addition to our results also data from structureactivity relationships of ring substituted dihydrochalcone sweeteners reported by Whitelaw et al. $[23,24]$ were taken into account. These studies reported that the hydroxyl group in the $\mathrm{B}$ ring system at $\mathrm{C} 3$ of 3'-carboxyhesperitin dihydrochalcone is essential for its sweet taste. Moreover, the introduction of a carboxylic acid at the $\mathrm{C} 3$ ' position in the A ring system enhances the sweetness of the dihydrochalcone structure. Projecting this knowledge onto the chemical structure of NHDC suggests that the $\mathrm{OH}$ group in the $\mathrm{C} 3$ position is important for receptor-ligand interactions and the $\mathrm{C} 3$ ' position should be in close proximity to H6413.33 (Fig. 3A). Furthermore, H641 3.33 was already proposed to interact with the carboxylic acid of lactisole [15] and the mutational studies of the present report indicate that the binding pockets of these two compounds do indeed overlap. The proposed binding mode of NHDC also suggests an interaction of the hydroxyl group at C3 with $\mathrm{S} \mathrm{CO}^{3.32}$ and the hydroxyl groups at $\mathrm{C}^{\prime}$ and $\mathrm{C} 2^{\prime}$ of NHDC could potentially interact with polar amino acids located in TM3 and TM7, such as H6413.33, Q6373.29, and C8017.39 respectively (Fig. 3A). In addition, favorable hydrophobic interactions between the $\mathrm{B}$ phenyl ring of NHDC could be envisioned with V62 $21^{2.58}$ and F6242.61, and between the linker of the $\mathrm{A}$ and $\mathrm{B}$ phenyl ring with L8007.38 and G8047.42. Furthermore, the model (Fig. 3A) predicts additional putative interaction sites, such as an interaction between the C3-hydroxylgroup of NHDC with $\mathrm{S} 620^{2.57}$ in TM2. Moreover, the polar sugar moieties of NHDC could be interacting with polar amino acids of TM4 and TM5, e.g. Y6994.60, T7245.37, R7255.38 and S7265.39.

\section{Validation of the model by functional analysis of additional mutants}

To verify the predictions of the model we selected 10 additional amino acids in hTAS1R3 (S6202.57, V6212.58, F6242.61, Y699 4.60, T7245.37, R7255.38, S726 ${ }^{5.39}$, L8007.38, C8017.39, and G8047.42) as candidates that might also influence the activation of the sweet receptor by NHDC.

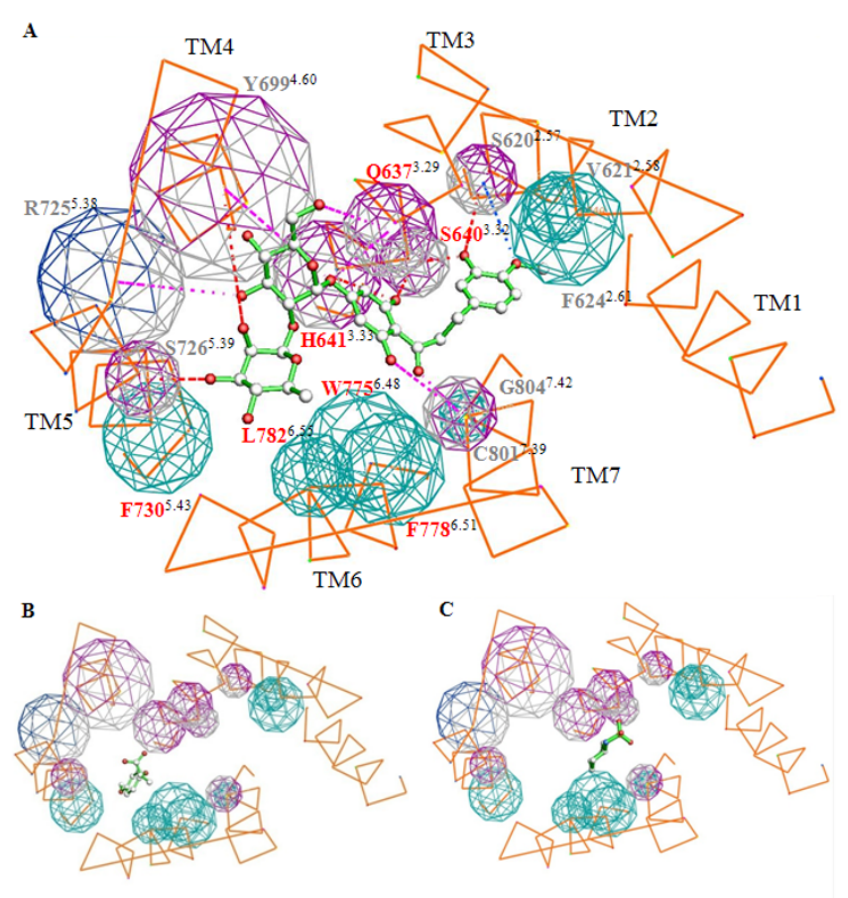

Figure 3

Three dimensional on top view of the TAS IR3 heptahelical domain binding pocket model. The binding pocket is docked with NHDC (A), lactisole (B), and cyclamate $(C)$. Intra- and extra cellular loops are removed and the helices are directly connected. The transmembrane segments (TMI-TM7) are denoted in orange. Important residues are labeled in Ballesteros-Weinstein nomenclature in addition to the one letter amino acid code. Amino acids that influence the activation of the sweet receptor by NHDC are shown as spheres. The size of the spheres corresponds to the size of the side chain. The spheres are colored according to their pharmacophoric properties. Hydrophobic amino acids ( $F, P$, M, A, L, I, G, V, W) cyan, H-donor/acceptor (Y, T, S, H, C, N, Q) magenta, $\mathrm{H}$-bond donors with a positive charge $(\mathrm{R})$ blue. Possible $\mathrm{H}$-bond interactions are presented as dotted lines The color of the dotted lines indicate the energy of a $\mathrm{H}$ bond, blue indicates an energy of $-0.3-0.6 \mathrm{kcal} / \mathrm{Mol}$, magenta: $-0.6-1.2 \mathrm{kcal} / \mathrm{Mol}$, red: - $1.2-2.4 \mathrm{kcal} / \mathrm{Mol}$. C atoms of NHDC are displayed in white and oxygen in red. Amino acid positions printed in red refer to residues emerged from the first round of mutational analysis and have been used as anchor points. Amino acid positions printed in grey were predicted from the model to influence receptor activation by NHDC and have been verified by mutational analysis.

To test their impact on the receptor function we created fourteen hTAS1R3 variants (S620A ${ }^{2.57}$, V621L ${ }^{2.58}$, V621I ${ }^{2.58}, \mathrm{~F} 624 \mathrm{~L}^{2.61}, \mathrm{Y} 699 \mathrm{~L}^{4.60}, \mathrm{Y} 699 \mathrm{~F}^{4.60}, \mathrm{~T} 724 \mathrm{~L}^{5.37}$,

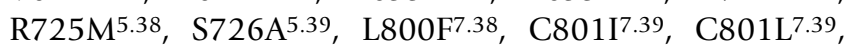
$\mathrm{G}^{2} 04 \mathrm{~A}^{7.42}$, and $\mathrm{G} 804 \mathrm{~V}^{7.42}$ ) and tested their responses to NHDC by calcium imaging after coexpression with hTAS1R2 in HEK293T-G16Gust44 cells. The results show 
that the mutant receptors V621L 2.58 and $\mathrm{C} 801 \mathrm{~L}^{7.39}$ could not be activated by NHDC, cyclamate, aspartame and acesulfame K (Table 2, Additional file 1), suggesting that these mutants are not functional. Therefore, we excluded them from further analyses. The receptor mutant L800F7.38 responded to NHDC in a manner similar to the wild type receptor (Table 2, Additional file 2), suggesting that L800 is not important for receptor activation by

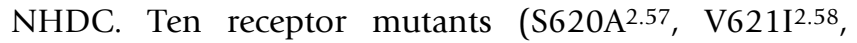

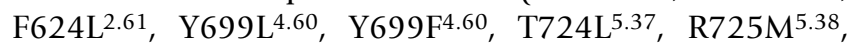
S726A 5.39 , C801I 7.39 , and G804A $\mathrm{A}^{7.42}$ ) had five to sixteen fold increased $\mathrm{EC}_{50}$ values for NHDC (Table 2, bold script, Additional file 2) and one mutant, G804V7.42, did not respond to NHDC at any tested concentration (Table 2, Additional file 1 and 2). The effects of these mutants were specific for NHDC because they all showed similar $\mathrm{EC}_{50}$ values for aspartame (Table 2). Moreover, the functional differences were not correlated with different expression levels or reduced signal amplitudes (Table 2, Additional file 3 ). The changes in the $\mathrm{EC}_{50}$ values suggest that these mutations directly affect the activation of the sweet taste receptor by NHDC, thus validating our model.

\section{Some of the newly identified residues also influence the interaction of the sweet receptor with lactisole or cyclamate}

Since our results indicate a partial overlap of the NHDC binding site with those for lactisole and cyclamate, we next asked, if any of the newly identified residues also affect the sweet receptor's responses to these compounds. To this end, we tested all mutant hTAS1R3 subunits by coexpression with hTAS1R2 in HEK293T-G16Gust44 cells for their sensitivity to lactisole and cyclamate (Additional file 1). While we essentially confirmed the findings of Jiang et al. $[14,15]$ that amino acids in positions $636^{3.28}$, $637^{3.29}, 640^{3.32}, 641^{3.33}, 721^{\mathrm{ex} 2}, 723^{5.36}, 729^{5.42}, 730^{5.43}$, $733^{5.46}, 778^{6.51}, 779^{6.52}, 782^{6.55}, 790^{\mathrm{ex} 3}$, and $798^{7.36}$ of hTAS1R3 contribute to the sensitivity of the sweet receptor to cyclamate and/or lactisole, we found additional amino acids in the heptahelical domain of hTAS1R3 that altered the responses of the sweet receptor to the two compounds. The mutant receptor $\mathrm{W} 775 \mathrm{~A}^{6.48}$ failed to respond to lactisole (Fig. 4A), suggesting that this residue is crucial for the sensitivity of the sweet receptor to the inhibitor. The mutants $\mathrm{C} 801 \mathrm{I}^{7.39}\left(\mathrm{IC}_{50}=0.3 \pm 0.01 \mathrm{mM}\right), \mathrm{Y} 99 \mathrm{~L}^{4.60}$ $\left(\mathrm{IC}_{50}=0.3 \pm 0.01 \mathrm{mM}\right)$, and $\mathrm{Y} 699 \mathrm{~F}^{4.60}\left(\mathrm{IC}_{50}=0.5 \pm 0.01\right.$ $\mathrm{mM}$ ) showed three- to fivefold reduced responses to lactisole compared to the wild type receptor $\left(\mathrm{IC}_{50}=0.1 \pm 0.01\right.$ $\mathrm{mM}$ ). Thus, we conclude that the amino acid positions Y699 4.60 and C8017.39 also contribute to the inhibition of the sweet receptor by lactisole. The mutants C801I ${ }^{7.39}$ and W775 $\mathrm{A}^{6.48}$ could not be activated at any tested concentration of cyclamate indicating the importance of these residues for the sweet receptor's responsiveness to cyclamate (Fig. 4B, Additional file 1). In addition, the mutant receptor $\mathrm{S} 726 \mathrm{~A}^{5.39}$ displayed more than fivefold lower sensitivity to cyclamate $\left(\mathrm{EC}_{50}>10 \mathrm{mM}\right)$ than the wild type receptor $\left(\mathrm{EC}_{50}=1.9 \pm 0.1 \mathrm{mM}\right)$, suggesting that all three residues contribute to the receptor's ability to be activated by cyclamate.

\section{Discussion}

NHDC binds in the heptahelical domain of TASIR3

Several independent lines of evidence clearly demonstrate that NHDC interacts with specific amino acid residues in the heptahelical domain of the TAS1R3 subunit. First, lac-

Table 2: Effect of mutations predicted by molecular modeling on the activation of the sweet taste receptor by neohesperidin dihydrochalcone (NHDC) and aspartame (ASP)

\begin{tabular}{|c|c|c|c|c|}
\hline variant & $\mathrm{NHDC} \mathrm{EC}_{50}(\mathrm{mM})$ & $\begin{array}{c}\text { NHDC maximal signal } \\
(\% \text { of } w t)\end{array}$ & ASP EC ${ }_{50}(\mathrm{mM})$ & $\begin{array}{c}\text { ASP maximal signal } \\
(\% \text { of } w t)\end{array}$ \\
\hline wt & $0.1 \pm 0.06$ & 100 & $1.3 \pm 0.1$ & 100 \\
\hline S620A 2.57 & $\mathrm{I} .3 \pm 0.5$ & $55 \pm 80$ & $1.2 \pm 0.3$ & $64 \pm 4$ \\
\hline $\mathrm{V} 62 \mathrm{IL}^{2.58}$ & n.f. & --- & n.f. & -- \\
\hline V62 I I $\left.\right|^{2.58}$ & $>1$ & $60 \pm 14$ & $1.9 \pm 0.4$ & $61 \pm 1$ \\
\hline F624L 2.61 & $>1$ & $35 \pm 70$ & $1.4 \pm 0.2$ & $21 \pm 4$ \\
\hline Y699L4.60 & $0.7 \pm 0.1$ & $83 \pm 40$ & $1.6 \pm 0.4$ & $78 \pm 9$ \\
\hline Y699F4.60 & $0.6 \pm 0.4$ & $72 \pm 17$ & $1.2 \pm 0.3$ & $56 \pm 2$ \\
\hline T724L5.37 & $0.5 \pm 0.3$ & $58 \pm 60$ & $1.5 \pm 0.2$ & $69 \pm 2$ \\
\hline$R^{2} 25 M^{5.38}$ & $1.3 \pm 0.4$ & $64 \pm 14$ & $1.5 \pm 0.7$ & $56 \pm 1$ \\
\hline S726A $\mathbf{A}^{5.39}$ & $0.9 \pm 0.3$ & $60 \pm 50$ & $0.9 \pm 0.3$ & $50 \pm 2$ \\
\hline L800F7.38 & $0.2 \pm 0.1$ & $91 \pm 70$ & $1.2 \pm 0.3$ & $90 \pm 1$ \\
\hline C80117.39 & $1.6 \pm 0.7$ & $70 \pm 13$ & $1.2 \pm 0.5$ & $50 \pm 9$ \\
\hline C80IL7.39 & n.f. & --- & n.f. & --- \\
\hline G804A $^{7.42}$ & $1.3 \pm 0.5$ & $53 \pm 10$ & $1.6 \pm 0.2$ & $44 \pm 1$ \\
\hline G804V7.42 & n.r. & --- & $1.3 \pm 0.3$ & $21 \pm 2$ \\
\hline
\end{tabular}

Receptor variants that affect the NHDC response are shown in bold. Values are given as average over at least three independent experiments \pm SD. n.f. no response to any tested sweetener, n.r. no response to neohesperidin dihydrochalcone up to highest tested concentration (6 mM), but responses to other sweeteners. $(>), \mathrm{EC}_{50}$ not calculable because saturation is not reached (Additional file 2 ). 

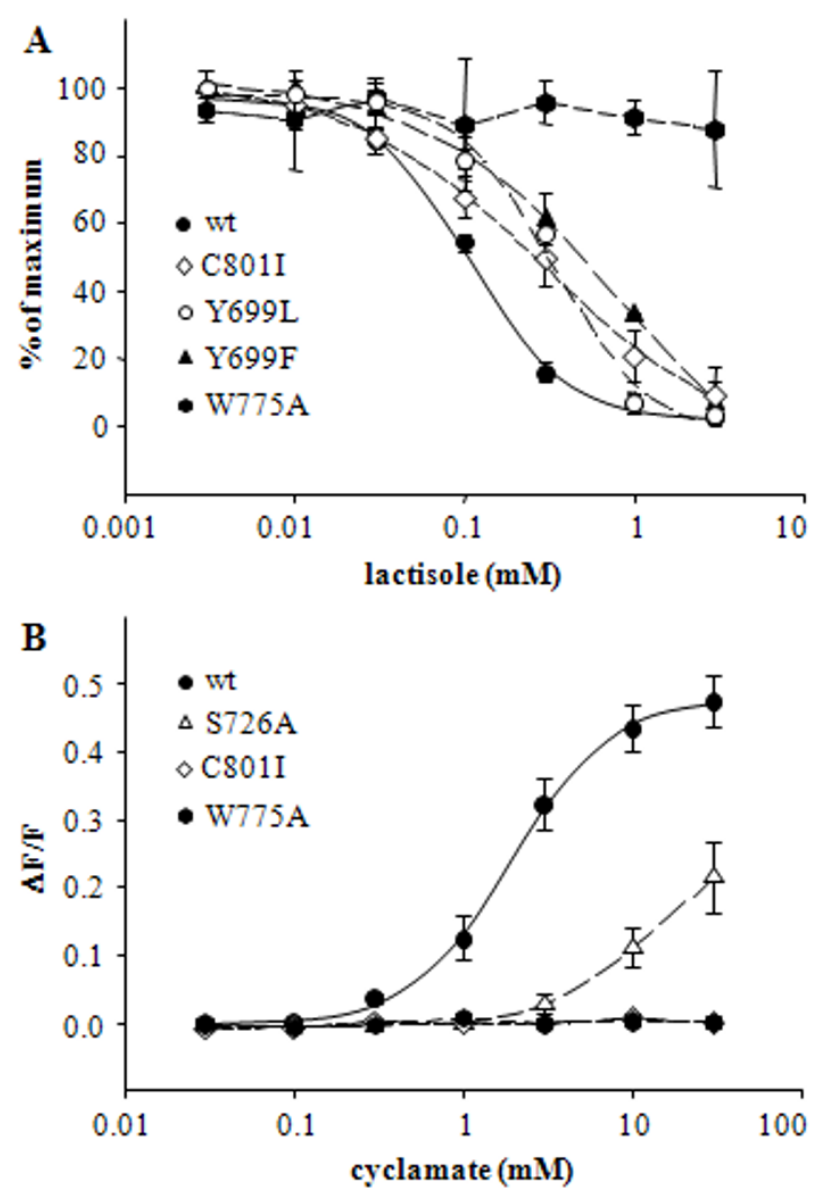

Figure 4

Identification of additional determinants in TAS IR3 critical for responsiveness to cyclamate and lactisole. (A) Concentration-dependent inhibition of calcium responses to $10 \mathrm{mM}$ aspartame by lactisole in HEK293T-G I6Gust44 cells cotransfected with DNA for wild type hTASIR2/ hTASIR3 (filled circle, solid line), C80117.39 (open diamond,

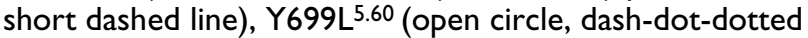
line), Y699 $\mathrm{F}^{5.60}$ (filled triangle up, medium dashed line), or W775A 6.48 (filled hexagon, dash-dotted line) and hTASIR2 DNA. (B) Concentration-dependent responses of HEK293TGI6Gust44 cells to cyclamate cotransfected with DNA for hTASIR3 (filled circle, solid line), S726 $\mathrm{A}^{5.39}$ (open triangle up, medium dashed line), C80 I17.39 (open diamond, short dashed line), or W775A 6.48 (filled hexagon, dash-dotted line) and hTASIR2.

tisole, which interacts with the heptahelical domain of TAS1R3 [13,15], inhibited the activation of hTAS1R2/ hTAS1R3 by NHDC competitively, while sweeteners that interact with other parts of the receptor were allosterically inhibited (Fig. 2). Second, the functional analysis of rat/ human TAS1R2/TAS1R3 chimeric receptors showed that NHDC activated all chimeric receptors that contain the heptahelical domain of human TAS1R3. In contrast, all chimeras that contained the heptahelical domain of rat Tas $1 \mathrm{r} 3$ could not be activated by NHDC (Fig. 1). Third, seventeen amino acid exchanges in the heptahelical domain of hTAS1R3 specifically reduced the NHDC responses but did not influence the responses to aspartame (Table $1+2$ ), which interacts with the extracellular $\mathrm{N}$-terminal domain of TAS1R2 [13]. Moreover, the finding that NHDC activates the human sweet receptor but not the rat sweet taste taste receptor delivers a molecular basis for the well-known lack of NHDC preference in rodents $[25,26]$. In addition, our results confirm previous findings that functional differences between rat and human sweet receptor [4] are caused by sequence variations in the heptahelical domain of TAS1R3 $[13,14,16]$.

\section{Elucidation of the NHDC binding site}

To further understand the interaction between NHDC and the heptahelical domain of hTAS1R3 we established a simple 3D receptor pharmacophore model of the NHDC binding site. Notably, in the model each amino acid residue in the heptahelical domain of the TAS1R3 receptor is identified by its position. Moreover, the numbering system proposed by Ballesteros and Weinstein [27] is shown as superscripts to facilitate the comparison with results of other GPCRs. The model considers results obtained from our functional studies, available data about the suggested binding modes of cyclamate and lactisole [14,15], information about conserved amino acids in GPCRs [27], and sensory studies of NHDC derivatives $[23,24]$. These sensory studies have shown that the hydroxyl group in C3 of the $\mathrm{B}$ ring is crucial for the sweet taste of dihydrochalcones $[23,28]$. In line with these findings, our receptor model proposes that this hydroxyl group can form hydrogen bonds with either of the two serine residues in positions $640^{3.32}$ of TM3 and $620^{2.57}$ of TM2. The possible interaction of the C3 hydroxyl group with both serines can explain why mutations at either position strongly reduced but not completely abolished the responses of the receptor to NHDC.

The model also predicts that H6413.33, Q6373.29, and C8017.39 can form hydrogen bonds with hydroxyl groups in the A ring as suggested by the decreased $\mathrm{EC}_{50}$ values for NHDC of the corresponding mutants. In addition, our model predicts that hydroxyl groups of the sugar moieties interact with Y6994.60, R7255.38, and S7265.39 in TM4 and TM5. Indeed, mutations in these positions impaired the interaction of NHDC with the receptor. Notably, the aglycon hesperitin dihydrochalcone that lacks the sugar rings also tastes sweet $[29,30]$. Therefore, it might be conceivable that amino acids at these three positions are less important for the activation of the sweet taste receptor by NHDC, but rather enhance its affinity. Furthermore, the methoxy group at position $\mathrm{C} 4$ of the $\mathrm{B}$ ring is in close proximity to V6212.58 and F6242.61 and is therefore likely 
engaged in favorable hydrophobic interactions with the side chains of these amino acids as concluded from the shift in $\mathrm{EC}_{50}$ values for NHDC at the corresponding mutant receptors.

\section{The binding sites for NHDC, cyclamate and lactisole share amino acid residues}

The sweet inhibitor lactisole, which interacts with specific residues in the heptahelical domain of TAS1R3 $[13,15,16]$, inhibited the activation of the sweet receptor by NHDC and cyclamate competitively (Fig. 2). These results suggest that the binding sites of lactisole, cyclamate, and NHDC overlap. Interestingly, the observed competitive effects between NHDC and lactisole (Fig. 2) may also explain why lactisole in contrast to many other sweeteners did not inhibit the sweet taste of NHDC in humans [31]. A previous report of Jiang et al. proposed that cyclamate and lactisole interact with a common set of amino acid residues[14,15]. Our findings confirm this observation by showing that seven mutations (Q6373.29, H6413.33, W7756.48, F7786.51, L782 ${ }^{6.55}$, R790Q ${ }^{\text {ex3 }}$, and C8017.39) influence both, the lactisole mediated inhibition and the cyclamate induced activation of the sweet receptor (Fig. 5, Table $1+2$ ). While cyclamate and lactisole only use parts of the TAS1R3 pharmacophore, our model predicts that NHDC uses most of it due to its larger size (Fig. 3). In line with these predictions, eight of the seventeen amino acids that alter receptor activation by NHDC (Q6373.29, S640 3.32, H641 3.33, Y6994.60, W7756.48, F7786.51, L7826.55, and C8017.39), also influence lactisolemediated inhibition of the receptor. Similarly, nine of the seventeen residues (Q6373.29, H6413.33, H721 ex2, S7265.39, F7305.43, W7756.48, F7786.51, L7826.55, and C8017.39) mediate activation by cyclamate, while six (Q6373.29, H6413.33, W7756.48, F7786.51, L7826.55, and C8017.39) influence receptor inhibition by lactisole as well as receptor activation by cyclamate (Fig. 5 , Table $1+2$ ).

Our mutational analysis identified four additional amino acid residues that have not previously been recognized (Y6994.60, S7265.39, W7756.48, and C8017.39) as interaction partners for lactisole and cyclamate $[14,15]$. These residues nicely refine the model proposed by Jiang et al. by enabling additional interactions between the two compounds and TAS1R3. This finding supports the validity of our model and suggests that it is compatible with that of Jiang et al.

Transmembrane domains six and seven are generally involved in GPCR activation [32-34]. The following observations suggest that the four residues $\mathrm{W} 775 \mathrm{~A}^{6.48}$, F778A 6.51 , L782A 6.55 , and C801I7.39 of TM6 and TM7 could participate in conformational changes of the sweet taste receptor from the ground to an active state and vice versa. Firstly, F7786.51 and W7756.48 are highly conserved

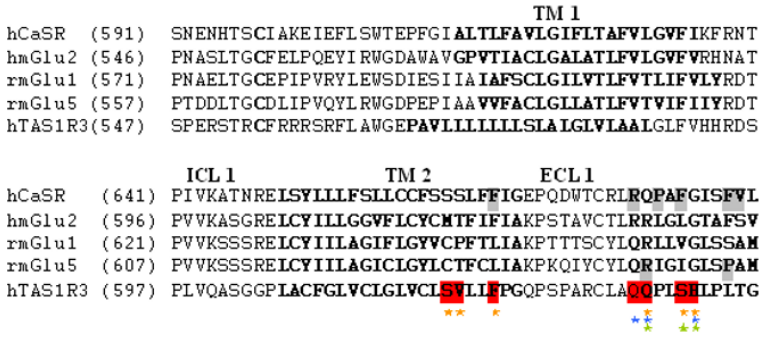

$\begin{array}{ccc}\text { TM } 3 & \text { ICL } 2 & \text { TM } 4 \\ \text { hCaSR (691) CISCIIVKTHRVLIVF EAKIPTSFHR--KWWGLINLOFLIVFLCTFHOIVI }\end{array}$ hCaSR (691) CISCILVKTNRV LIVF EAK IPTSF HR--KWWGL NLOFLIVFLCTFHQIVI
hMGlu2 hmGlu2 (646) CYSAILTKTNRI ARIF GGAREGAQR--PRF ISP ASQVAICI.ALISGQIII
rmGlu1 (671) CYSAIVTKTNRI ARIL AGSKKKICTRKPRFMSA WAQVIIASILISVQLTI rMGlu5 (657) SYSALVTKTNRI ARIL AGSKKKICTKKPRF MSA CAQLVIAFILICIQLGI hTAS1R3 (647) CISTIFLQAAEI FVESELPLSWADR-ISGCLRGPUAVIVVLLAHIVEVAI

ECL 2

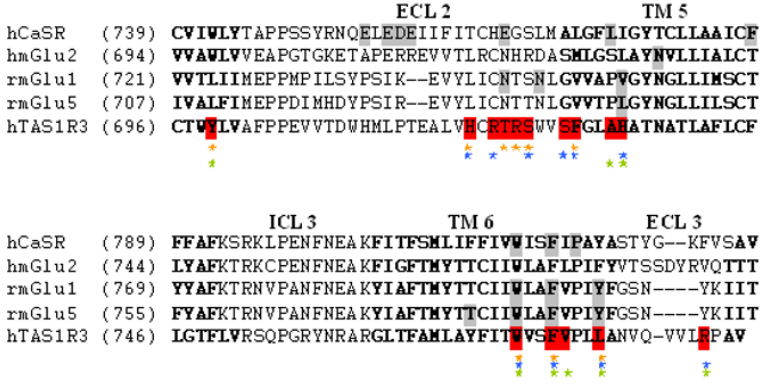

TM 7

hCaSR (837) EVIAILAASFGLIACIFFNKIYI I LF KPSRNT I EEVRCST AAHAFKV AAR hMG1 u2 (794) WCVSVSISGSWVLGCIFAP KLHI I LFQPQKNVVSHRAPTSRFGSAAARAS rmGlu 1 (794) TCFAVSISVTVALGCHFTPKMY I I IA KPERNVRSAFT TSDVVRM HVGDG $r m G 1 u 5$ (801) HCFSVSISA TVALGCHFVP KVYI I LAKPERNVRSAFT TSTVVRM HVGDGK hTAS1R3 (794) QHGAMILCVIGILAAFHIPRCYLIMROPGLNTPEFFIGGG PDAQGQNDG 菜

\section{Figure 5}

Alignment of the heptahelical domain of hCaSR, hmGlu2, rmGlu I, rmGlu5, and hTASIR3. Transmembrane segments (TM) are shown in bold. Intra- and extracellular loops are marked by ICL and ECL, respectively. Amino acids that influence allosteric modulator activity in hCaSR, hmGlu2, rmGlul and 5 are marked in grey. Positions that alter the response of the sweet receptor to lactisole, cyclamate, or NHDC are shown in red. Asterisks indicate residues involved in receptor activation by NHDC (orange) or cyclamate (blue). Green asteriks denote residues mediating sensitivity of the receptor to lactisole.

across class A and class C GPCRs [14,15,35]. Secondly, our study showed that mutations at these positions alter the receptor's sensitivities to the agonists NHDC and cyclamate as well as to the inhibitor lactisole. Thirdly, Jiang et al. also found that mutations in position L782 $\mathrm{A}^{6.55}$ abolished activation of the sweet taste receptor by cyclamate and enhanced the receptor's responsiveness to the inhibitor lactisole [15].

The amino acid residue C8017.39 in TM7 also affects the sensitivity of the sweet taste receptor to cyclamate, NHDC and lactisole. While it is in the proximity of the NHDC 
and cyclamate binding sites, it is quite remote from that of lactisole (Fig. 3A, B, C). This argues against a direct interaction of lactisole with C8017.39. It is therefore conceivable that in addition to W775 $5^{6.48}, \mathrm{~F} 778^{6.51}$, and $\mathrm{L} 782^{6.55}$ in TM6, C8017.39 in TM7 may also play a general role in the activation process of the sweet taste receptor. This assumption is further supported by the observation that amino acids in TM7 of the calcium sensing receptor such as $\mathrm{V} 836^{7.31}$, Q837 7.32 and $\mathrm{A} 843^{7.38}$ play a key role in the activation of this receptor [32].

Notably, aspartame, which likely interacts with the N-terminal domain of hTAS1R2 $[12,13]$ could activate the mutants W7756.48, F7786.51, L7826.55 and C801I ${ }^{7.39}$ in a manner, similar to the wild type receptor (Tab. 1 and 2). Therefore, it might be possible that tastants that bind to different receptor sites elicit different types of active confirmations as has been observed before for the activation of the $\beta_{2}$-adrenergic receptor by salbutamol and catechol [36].

\section{The NHDC binding site overlaps with the binding sites of allosteric modulators in other class C GPCRs}

Sequence comparisons revealed that nine of the amino acid positions in hTAS1R3 that influence the sensitivity of the sweet receptor to NHDC, cyclamate or lactisole correspond to residues that are responsible for binding allosteric modulators in the calcium sensing receptor and metabotropic glutamate receptors (Fig. 5) [32-34,37-43]. This suggests a general role for these positions in allosterism in this class of receptors and shows that positions of allosteric binding sites are partly conserved amongst class C GPCRs. Thus, critical determinants found in non-taste receptors are good candidates to uncover additional binding sites for sweet and umami tasting substances or modulators.

\section{Conclusion}

Our mutational analysis combined with functional studies and molecular modeling identified the binding pocket of NHDC in the heptahelical domain of human TAS1R3. We found that the binding pocket of NHDC overlaps with those of the sweetener cyclamate and the sweet taste inhibitor lactisole. Seven of the amino acid positions crucial for activation of hTAS1R2/hTAS1R3 by neohesperidin dihydrochalcone are involved in the binding of allosteric modulators of other class C GPCRs. This suggests a general role of these amino acid positions in allosterism and points to a common architecture of the heptahelical domains of class C GPCRs.

\section{Methods}

\section{Ballesteros and Weinstein nomenclature}

The position of each amino acid residue in the heptahelical domain of TAS1R3 is identified both by its position and by the generic numbering system proposed by Ballesteros and Weinstein [27] shown as superscripts. In the Ballesteros-Weinstein nomenclature the most conserved residue in each helix is given the number 50 . This is N1.50, D2.50, R3.50, W4.50, P5.50, P6.50 and P7.50 in transmembrane helix 1-7 of the rhodopsin receptor. The corresponding amino acids in the TAS1R3 are given the same numbers (Additional file 4).

\section{Construction of TASIR3 mutants}

Human TAS1R3 receptor mutants were generated by site directed mutagenesis according to the QuickChange protocol (Stratagene, La Jolla, CA). The forward and reverse primers contained the desired mutations and annealed to the same sequence on opposite strands of the plasmids. The following TAS1R3 receptor mutants were generated: S620A 2.57, V621L $L^{2.58}$, V621I $2.58, F_{624 L} 2.61$, Q636A 3.28 ,

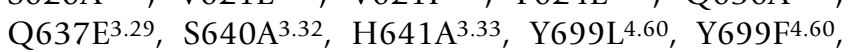

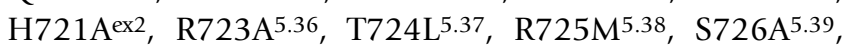
S729A ${ }^{5.42}$, F730L 5.43, A733V5.46, H734A ${ }^{5.47}$, W775A 6.48 ,

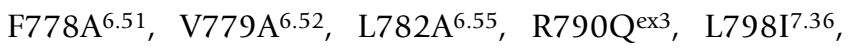
L800F7.38, C801L7.39, C80117.39, G804V7.42, and G804A 7.42 .

The position of each amino acid residue in the seven heptahelical domain of the TAS1R3 receptor is identified both by its position and by the generic numbering system proposed by Ballesteros and Weinstein [27] shown as superscripts.

\section{Functional expression}

The cDNAs for TAS1Rs tagged at the C-terminus with the herpes simplex virus glycoprotein D (HSV) were transiently transfected into HEK293T cells stably expressing the chimeric G-protein subunit G16Gust44 [44] using Lipofectamine 2000 (Invitrogen, Carlsbad, CA) according to the manufacturer's protocol. 3-4 hours after transfection, DMEM was replaced by low-glucose DMEM supplemented with GlutaMAX and 10\% dialyzed FBS (Invitrogen, Carlsbad, CA). $24 \mathrm{~h}$ post transfection, cells were loaded for $1 \mathrm{~h}$ with the calcium sensitive dye Fluo4AM $(2 \mu \mathrm{g} / \mathrm{ml}$ in DMEM, Molecular Probes, Carlsbad, CA). Cells were washed $3 \mathrm{x}$ in solution $\mathrm{C} 1(130 \mathrm{mM} \mathrm{NaCl}, 5$ $\mathrm{mM} \mathrm{KCl}, 10 \mathrm{mM}$ Hepes, $2 \mathrm{mM} \mathrm{CaCl}_{2}$, and $5 \mathrm{mM}$ Glucose, $\mathrm{pH} 7,4)$. We monitored calcium mobilization following receptor stimulation with sweet tastants by an automated fluorometric imaging plate reader (FLIPR, Molecular Devices, Sunnyvale, CA). Compounds used as stimuli (Sigma-Aldrich, St. Louis, MO; Merck, Whitehouse Station, NJ) were dissolved in C1 solution. All data were collected from at least three independent experiments carried out in duplicate. The obtained calcium signals were corrected for the response of mock transfected cells and normalized to the fluorescence of cells prior to the application of the stimulus using $\Delta \mathrm{F} / \mathrm{F}=(\mathrm{F}-\mathrm{F} 0) / \mathrm{F} 0$. Con- 
centration-response curves and $\mathrm{EC}_{50}$ and $\mathrm{IC}_{50}$ values were calculated in SigmaPlot by nonlinear regression using the function $\mathrm{f}=\left((\mathrm{a}-\mathrm{d}) /\left(1+(\mathrm{x} / \mathrm{EC} 50)^{\mathrm{nH}}\right)+\mathrm{d}\right)$ and $\mathrm{f}=(\mathrm{a}-\mathrm{b}) /$ $\left[1+(\mathrm{x} / \mathrm{IC} 50)^{n \mathrm{H}}\right]+\mathrm{b}$, respectively.

\section{Immuncytochemistry}

HEK293T-G16Gust44 cells were seeded on poly-D-lysine coated coverslips $(10 \mu \mathrm{g} / \mathrm{ml})$ and transfected with the respective cDNAs. $24 \mathrm{~h}$ post transfection cells were washed with PBS and fixed and permeabilized for $5 \mathrm{~min}$ in acetone/methanol (1:1). Incubating the cells in 5\% goat serum for 30 min reduced non-specific binding. To detect the receptors, antiserum against the HSV-epitope (mouse anti-HSV (Novagen, Madison, WI), 1:10000 in $3 \%$ goat serum) was added to the cells for $1 \mathrm{~h}$ at room temperature (RT). After washing the cells three times with PBS we added Alexa488-conjugated goat antiserum against mouse IgG (Molecular Probes, Carlsbad, CA), $1: 1000$ in $3 \%$ goat serum) for $1 \mathrm{~h}$ at RT. The cells were embedded in Fluorescent Mounting Medium (Dako, Glostrup, Denmark) and analyzed using a fluorescence microscope (Zeiss Axioplan, Jena, Germany) and a camera (RT Slide, Visitron Systems, Munich, Germany).

\section{TASIR3 modeling}

All modeling calculations were made on a Silicon Graphics Octane with a single R12000 processor using our inhouse modeling package Moloc [45]. The alignment of the seven transmembrane helices of TAS1R3 receptor (SWISS-PROT: TS1R3_HUMAN, Q7RTX0) with the transmembrane helices of bovine rhodopsin (pdb ref code $1 \mathrm{f88}$ ) and the 3D receptor-based pharmacophore modeling was described previously [22]. Briefly, the method relies on a robust alignment algorithm based on conservation indices, focusing on pharmacophore-like relationships between amino acids. Analysis of conservation patterns across the GPCR family and alignment to the rhodopsin $\mathrm{x}$-ray structure (pdb ref code $1 \mathrm{f} 88$, Additional file 4) allows the extraction of the amino acids lining the TM binding pocket in a so-called ligand binding pocket vector. In a second step, LPVs are translated to simple 3D receptor pharmacophore models, where a single spherical pharmacophore feature represents each amino acid and all atomic detail is omitted. The pharmacophores are colored according to the pharmacophoric properties of the amino acids. Hydrophobic amino acids (aromatic/ aliphatic; F, P, M, A, L, I, G, V, W) are represented by cyan pharmacophores, amino acids with H-donor/acceptor functionalities (Y, T, S, H, C, N, Q) by magenta pharmacophores, and amino acids with $\mathrm{H}$-bond donor functionalities and positive charge $(\mathrm{K}, \mathrm{R})$ by blue ones. The pharmacophores with H-bond donor or acceptor properties have in addition a cone, which gives the most likely direction of the H-bond. The new reported receptor-based pharmacophore modeling methodology allows a visual analysis of the general ligand binding properties of any GPCR without the need to concentrate on atomic details of side chain orientations [22]. NHDC is manually docked into the pharmacophores in the TM pocket. Only pharmacophores are shown which were identified as important for the action of NHDC by site-directed mutagenesis.

\section{Abbreviations \\ NHDC - neohesperidin dihydrochalcone \\ GPCR - G-protein coupled receptor \\ TAS1R - taste receptor family one}

HEK293T-G16Gust44 - human embryonal kidney cell line stably expressing the large $\mathrm{T}$ antigen and the G-protein chimera G16Gust 44

$\mathrm{EC}_{50}-$ half maximal effective concentration

$\mathrm{IC}_{50}-$ half maximal inhibitory concentration

FLIPR - fluorometric imaging plate reader

\section{Competing interests}

The author(s) declares that there are no competing interests.

\section{Authors' contributions}

MW cloned most of the TAS1R subunits, constructed the chimeric TAS1R3 receptors, carried out the functional and histological studies and participated in the study design. $\mathrm{BB}$ coordinated the study design, hold and organized the external collaborations and drafted the manuscript. NAK did the molecular modeling and suggested several sites for receptor mutation. JPS participated in the study design. WM conceived the study, participated in its design, and helped to draft the manuscript. All authors read and approved the final manuscript.

\section{Additional material}

\section{Additional file 1}

Activity of all mutants towards different sweeteners.

Click here for file

[http://www.biomedcentral.com/content/supplementary/14726807-7-66-S1.tiff]

\section{Additional file 2}

Concentration-response curves of all mutants towards NHDC. Click here for file

[http://www.biomedcentral.com/content/supplementary/14726807-7-66-S2.pdf] 


\section{Additional file 3 \\ Expression of hTAS1R3 variants. \\ Click here for file \\ [http://www.biomedcentral.com/content/supplementary/1472- 6807-7-66-S3.tiff]}

\section{Additional file 4}

Sequence alignment of human TAS1R3 and bovine rhodopsin transmembrane segments (TM).

Click here for file

[http://www.biomedcentral.com/content/supplementary/14726807-7-66-S4.tiff]

\section{Acknowledgements}

The authors gratefully acknowledge Stefanie Demgensky, and Ellen Schöley Pohl for expert technical assistance. We thank Dietmar Krautwurst for his helpful comments and discussion. We are grateful to Hartwig Schmale for the rat Tas $I r 3$ cDNA. This research was supported in part by the German Science Foundation (ME 1024/I-2 and ME 1024/2-1).

\section{References}

I. Montmayeur JP, Liberles SD, Matsunami H, Buck LB: A candidate taste receptor gene near a sweet taste locus. Nat Neurosci 200I, 4:492-498.

2. Damak S, Rong M, Yasumatsu K, Kokrashvili Z, Varadarajan V, Zou S jiang P, Ninomiya $Y$, Margolskee RF: Detection of sweet and umami taste in the absence of taste receptor TIr3. Science 2003, 30I:850-853.

3. Hoon MA, Adler E, Lindemeier J, Battey JF, Ryba NJ, Zuker CS: Putative mammalian taste receptors: a class of taste-specific GPCRs with distinct topographic selectivity. Cell 1999, 96:54I-55I.

4. Li X, Staszewski L, Xu H, Durick K, Zoller M, Adler E: Human receptors for sweet and umami taste. Proc Natl Acad Sci U S A 2002, 99:4692-4696

5. Nelson G, Hoon MA, Chandrashekar J, Zhang Y, Ryba NJ, Zuker CS Mammalian sweet taste receptors. Cell 200I, I 06:38I-390.

6. Max M, Shanker YG, Huang L, Rong M, Liu Z, Campagne F, Weinstein $H$, Damak S, Margolskee RF: Tas Ir3, encoding a new candidate taste receptor, is allelic to the sweet responsiveness locus Sac. Nat Genet 200I, 28:58-63.

7. Terrillon S, Bouvier M: Roles of G-protein-coupled receptor dimerization. EMBO Rep 2004, 5:30-34.

8. Nelson G, Chandrashekar J, Hoon MA, Feng L, Zhao G, Ryba NJ, Zuker CS: An amino-acid taste receptor. Nature 2002, 416: 199-202.

9. Brouwer JN, Hellekant G, Kasahara $Y$, van der Wel H, Zotterman $Y$ : Electrophysiological study of the gustatory effects of the sweet proteins monellin and thaumatin in monkey, guinea pig and rat. Acta Physiol Scand 1973, 89:550-557.

10. Sclafani $A, A b r a m s$ M: Rats show only a weak preference for the artificial sweetener aspartame. Physiol Behav 1986, 37:253-256.

II. Sclafani A, Perez C: Cypha [propionic acid, 2-(4-methoxyphenol) salt] inhibits sweet taste in humans, but not in rats. Physiol Behav 1997, 6I:25-29.

12. Jiang P, Ji Q, Liu Z, Snyder LA, Benard LM, Margolskee RF, Max M: The cysteine-rich region of TIR3 determines responses to intensely sweet proteins. I Biol Chem 2004, 279:45068-45075.

13. Xu H, Staszewski L, Tang H, Adler E, Zoller M, Li X: Different functional roles of TIR subunits in the heteromeric taste receptors. Proc Natl Acad Sci U S A 2004, I 1 I: | 4258- | 4263.

14. Jiang P, Cui M, Zhao B, Snyder LA, Benard LM, Osman R, Max M, Margolskee RF: Identification of the cyclamate interaction site within the transmembrane domain of the human sweet taste receptor subunit TIR3. I Biol Chem 2005, 280:34296-34305.
15. Jiang P, Cui M, Zhao B, Liu Z, Snyder LA, Benard LM, Osman R, Margolskee RF, Max M: Lactisole interacts with the transmembrane domains of human TIR3 to inhibit sweet taste. J Biol Chem 2005, 280:15238-15246.

16. Winnig M, Bufe B, Meyerhof $W$ : Valine $\mathbf{7 3 8}$ and lysine $\mathbf{7 3 5}$ in the fifth transmembrane domain of $r T$ as $1 r 3$ mediate insensitivity towards lactisole of the rat sweet taste receptor. $B M C$ Neurosci 2005, 6:22

17. Dogan M: Neohesperidin DC in food products: ; Trabzon, Türkiye. Volume I.; 2002:190-195.

18. DuBois GE, Crosby GA, Stephenson RA, Wingard RE Jr.: Dihydrochalcone sweeteners. Synthesis and sensory evaluation of sulfonate derivatives. J Agric Food Chem 1977, 25:763-772.

19. DuBois GE, Crosby GA, Stephenson RA: Dihydrochalcone sweeteners. A study of the atypical temporal phenomena. J Med Chem 1981, 24:408-428.

20. Durroux T: Principles: a model for the allosteric interactions between ligand binding sites within a dimeric GPCR. Trends Pharmacol Sci 2005, 26:376-384.

21. Morini G, Bassoli A, Temussi PA: From small sweeteners to sweet proteins: anatomy of the binding sites of the human TIR2_TIR3 receptor. J Med Chem 2005, 48:5520-5529.

22. Kratochwil NA, Malherbe P, Lindemann L, Ebeling M, Hoener MC Muhlemann A, Porter RH, Stahl M, Gerber PR: An automated system for the analysis of $\mathbf{G}$ protein-coupled receptor transmembrane binding pockets: alignment, receptor-based pharmacophores, and their application. J Chem Inf Model 2005, 45: 1324-1336.

23. Whitelaw ML, Chung HJ, Daniel JR: Synthesis and sensory evaluation of ring-substituted dihydrochalcone sweeteners. 2. Analogues of 3'-Carboxyhesperetin dihydrocahlcone, a highpotency dihydrochalcone sweetener. J Agric Food Chem 1991, 39:663-667.

24. Whitelaw ML, Daniel JR: Synthesis and sensory evaluation of ring-substituted dihydrochalcone sweeteners. J Agric Food Chem 1991, 39:44-51.

25. Naim M, Rogatka $H$, Yamamoto $T$, Zehavi U: Taste responses to neohesperidin dihydrochalcone in rats and baboon monkeys. Physiol Behav 1982, 28:979-986.

26. Bachmanov AA, Tordoff MG, Beauchamp GK: Sweetener preference of C57BL/6ByJ and I29P3/J mice. Chem Senses 200I, 26:905-913

27. Ballesteros JA, H. W: Integrated Methods for the Construction of Three-Dimensional Models and Computational Probing of Structure-Function Relations in G-Protein-Coupled Receptors. Methods in Neuroscience 1995, 25:366-428.

28. Bassoli A, Merlini L, Morini G: Isovanillyl sweeteners. From molecules to receptors. Pure Appl Chem 2002, 74: I I8I-I I87.

29. DuBois GE, Crosby GA, Saffron P: Nonnutritive sweeteners: taste-structure relationships for some new simple dihydrochalcones. Science 1977, 195:397-399.

30. Horowitz RM, Gentili B: Flavonoids of the Ponderosa lemon. Nature 1960, 185:319.

3I. Schiffman SS, Booth BJ, Sattely-Miller EA, Graham BG, Gibes KM: Selective inhibition of sweetness by the sodium salt of $+/-2$ (4-methoxyphenoxy)propanoic acid. Chem Senses 1999, 24:439-447.

32. Hu J, McLarnon SJ, Mora S, Jiang J, Thomas C, Jacobson KA, Spiegel AM: A region in the seven-transmembrane domain of the human $\mathrm{Ca2}+$ receptor critical for response to $\mathrm{Ca2+}$. J Biol Chem 2005, 280:5113-5120.

33. Malherbe P, Kratochwil N, Knoflach F, Zenner MT, Kew JN, Kratzeisen C, Maerki HP, Adam G, Mutel V: Mutational analysis and molecular modeling of the allosteric binding site of a novel, selective, noncompetitive antagonist of the metabotropic glutamate I receptor. J Biol Chem 2003, 278:8340-8347.

34. Petrel C, Kessler A, Maslah F, Dauban P, Dodd RH, Rognan D, Ruat $M$ : Modeling and mutagenesis of the binding site of Calhex $23 \mathrm{I}$, a novel negative allosteric modulator of the extracellular Ca(2+)-sensing receptor. J Biol Chem 2003, 278:49487-49494.

35. Pin JP, Galvez T, Prezeau L: Evolution, structure, and activation mechanism of family 3/C G-protein-coupled receptors. Pharmacol Ther 2003, 98:325-354.

36. Swaminath G, Deupi X, Lee TW, Zhu W, Thian FS, Kobilka TS, Kobilka B: Probing the beta2 adrenoceptor binding site with 
catechol reveals differences in binding and activation by agonists and partial agonists. J Biol Chem 2005, 280:22I65-22I7I.

37. Hu J, Reyes-Cruz G, Chen W, Jacobson KA, Spiegel AM: Identification of acidic residues in the extracellular loops of the seventransmembrane domain of the human $\mathrm{Ca} 2+$ receptor critical for response to $\mathrm{Ca2}+$ and a positive allosteric modulator. J Biol Chem 2002, 277:46622-4663।.

38. Malherbe P, Kratochwil N, Zenner MT, Piussi J, Diener C, Kratzeisen C, Fischer C, Porter RH: Mutational analysis and molecular modeling of the binding pocket of the metabotropic glutamate 5 receptor negative modulator 2-methyl-6-(phenylethynyl)-pyridine. Mol Pharmacol 2003, 64:823-832.

39. Miedlich SU, Gama L, Seuwen K, Wolf RM, Breitwieser GE: Homology modeling of the transmembrane domain of the human calcium sensing receptor and localization of an allosteric binding site. J Biol Chem 2004, 279:7254-7263.

40. Pagano A, Ruegg D, Litschig S, Stoehr N, Stierlin C, Heinrich M, Floersheim P, Prezeau L, Carroll F, Pin JP, Cambria A, Vranesic I, Flor PJ, Gasparini $F$, Kuhn R: The non-competitive antagonists 2methyl-6-(phenylethynyl)pyridine and 7-hydroxyiminocyclopropan[b]chromen-I a-carboxylic acid ethyl ester interact with overlapping binding pockets in the transmembrane region of group I metabotropic glutamate receptors. J Biol Chem 2000, 275:33750-33758.

4I. Petrel C, Kessler A, Dauban P, Dodd RH, Rognan D, Ruat M: Positive and negative allosteric modulators of the $\mathrm{Ca2+}$-sensing receptor interact within overlapping but not identical binding sites in the transmembrane domain. J Biol Chem 2004, 279: $18990-18997$

42. Ray K, Tisdale J, Dodd RH, Dauban P, Ruat M, Northup JK: Calindol, a positive allosteric modulator of the human $\mathrm{Ca}(2+)$ receptor, activates an extracellular ligand-binding domain-deleted rhodopsin-like seven-transmembrane structure in the absence of $\mathrm{Ca}(2+)$. I Biol Chem 2005, 280:370I3-37020.

43. Schaffhauser $H$, Rowe BA, Morales $S$, Chavez-Noriega LE, Yin R, Jachec C, Rao SP, Bain G, Pinkerton AB, Vernier JM, Bristow LJ, Varney MA, Daggett LP: Pharmacological characterization and identification of amino acids involved in the positive modulation of metabotropic glutamate receptor subtype 2. Mol Pharmacol 2003, 64:798-810.

44. Ueda T, Ugawa S, Yamamura H, Imaizumi Y, Shimada S: Functional interaction between T2R taste receptors and G-protein alpha subunits expressed in taste receptor cells. I Neurosci 2003, 23:7376-7380.

45. Molecular design software [http://www.moloc.ch] http.//www.biomedcentral.com/1472-6807/7/66 